\title{
Regulation of Cytoplasmic Dynein by Lis1
}

Katerina Toropova ${ }^{1 *}$, Sirui Zou ${ }^{2 *}$, Anthony J. Roberts ${ }^{2,3}$, Samara L. Reck-Peterson ${ }^{2 \#}$ and Andres E. Leschziner $^{1 \#}$

${ }^{1}$ Department of Molecular and Cellular Biology, Harvard University, Cambridge, MA, 02138, USA

${ }^{2}$ Department of Cell Biology, Harvard Medical School, Boston, MA, 02115

${ }^{3}$ Astbury Centre for Structural Molecular Biology, School of Molecular and Cellular Biology, Faculty of Biological Sciences, University of Leeds, Leeds, LS2 9JT, UK.

*These authors contributed equally to this work; \#Co-corresponding authors

Regulation of cytoplasmic dynein's motor activity is essential for diverse eukaryotic functions, including cell division, intracellular transport and brain development. The dynein regulator Lis1 is known to keep dynein bound to microtubules; however, how this is accomplished mechanistically remains unknown. We used three-dimensional (3D) electron microscopy (EM) and single-molecule imaging to help establish this mechanism. The 3D structure of the dynein-Lis1 complex shows that binding of Lis1 to dynein's AAA+ ring sterically prevents dynein's main mechanical element, the "linker", from completing its normal conformational cycle. Single-molecule experiments show that eliminating this block by shortening the linker to a point where it can physically bypass Lis1 renders single dynein motors insensitive to regulation by Lis1. Our data reveal that Lis1 keeps dynein in a persistent microtubule-bound state by directly blocking the progression of its mechanochemical cycle.

Cytoplasmic dynein 1 (“dynein” here) is a microtubule (MT)-based motor that moves towards the minus ends of MTs (generally towards the cell center). Dynein is a member of the AAA+ (ㅅTPases $\underline{\text { Associated }}$ with various cellular Activities) superfamily [1] and its motor contains a ring of six concatenated AAA+ domains (termed AAA1-6). The dynein regulator Lis1 was first described as the gene mutated in patients with type-1 lissencephaly, a neurodevelopmental disease characterized by a smooth cerebral surface, cognitive defects, and seizures [2, 3]. Biochemical experiments suggest that Lis1 increases dynein’s affinity for MTs [4-6]. Here, we set out to determine how Lis1 does this.

To determine the mechanism of dynein regulation by Lis1 we used both cryo-negative stain EM to determine structures of dynein-Lis1 complexes and single-molecule fluorescence microscopy to test hypotheses derived from our structural studies.

Our 3D EM structure showed that Lis1 binds at AAA4 in dynein and repositions dynein's main mechanical element, the linker domain (Figure 1) [7]. We hypothesized that Lis1 binding to dynein blocks the linker from reaching a key docking site at AAA5, an interaction that is required to release dynein from MTs. To test this idea, we made a mutation in dynein that we predicted would both allow Lis1 to bind dynein, as well as allow the linker to reach its AAA5 docking site. We confirmed that this was the case be determining EM structures of the dynein mutant bound to Lis1. Next we performed single-molecule experiments with this "short linker" mutant and found that the mutant was now insensitive to Lis1 binding. The short linker dynein no longer remained tightly bound to MTs in the presence of Lis1 and ATP (Figure 2) [7]. Together our results suggest that Lis1 keeps dynein in a persistent MT-bound state by directly blocking the progression of its mechanochemical cycle [7]. 


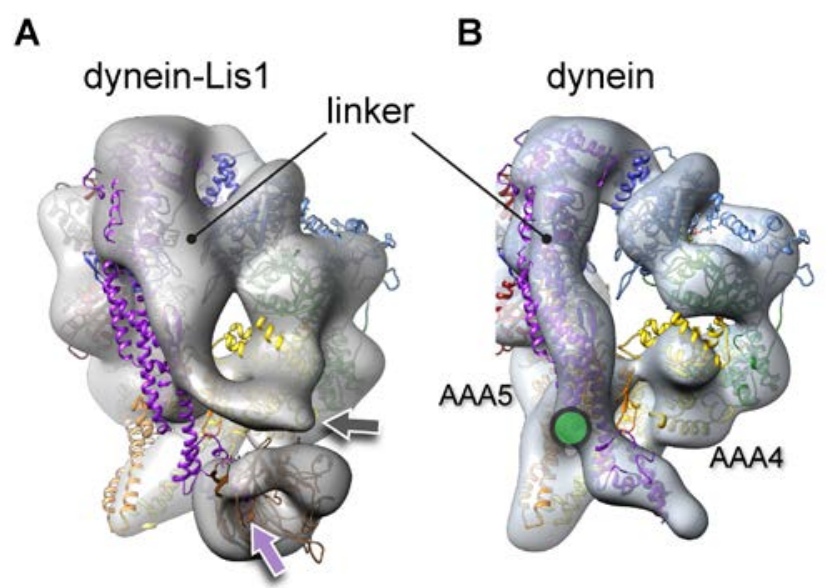

Figure 1. Structure of the dynein-Lis1 complex. (A) Structural model of dynein's motor domain (PDB ID: 4AKG) docked into the EM map of dynein-Lis1 (resolution $21 \AA$ ). The linker (grey arrow) is shifted away from its position in the crystal structure (purple arrow). (B) Structural model of dynein's motor domain docked into the EM map of dynein alone (resolution $15 \AA$ ). Green circle: location of linker interaction with AAA5.

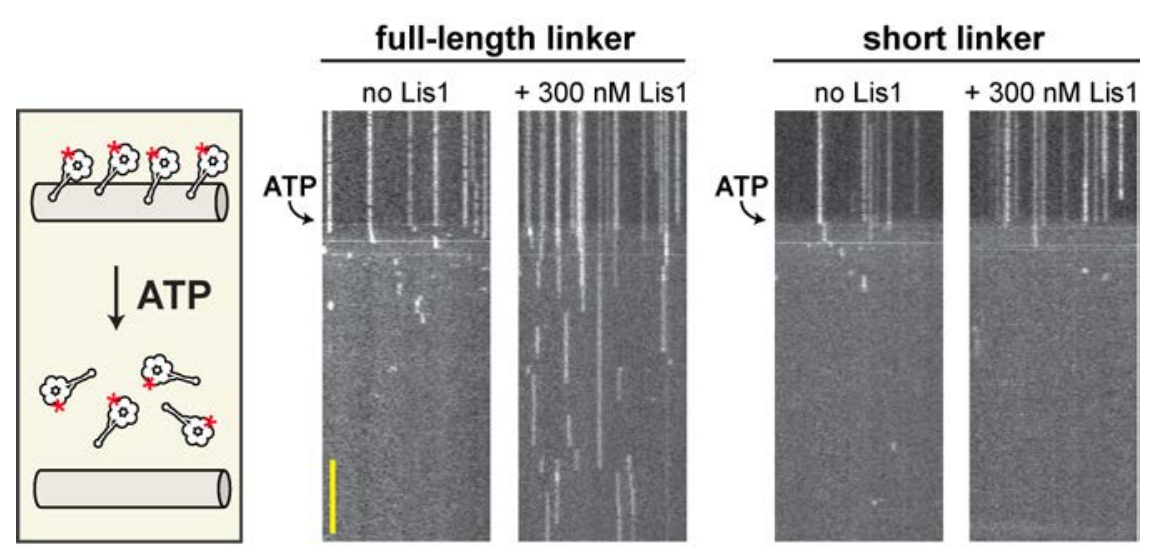

Figure 2. Short linker dynein is insensitive to Lis1. Diagram of the single-molecule release assay (left). TMR-labelled (red star) dynein molecules release from MTs in the presence of ATP. Lis1 causes dynein to remain bound to MTs (middle), while the short linker dynein is insensitive to Lis1 (right). Scale bar = 5 seconds.

\section{References}

[1] Neuwald, A.F., et al., Genome Res, 1999. 9(1): p. 27-43.

[2] Reiner, O., et al., Nature, 1993. 364(6439): p. 717-21.

[3] Wynshaw-Boris, A., Clin Genet, 2007. 72(4): p. 296-304.

[4] Huang, J., et al., Cell, 2012. 150(5): p. 975-86.

[5] McKenney, R.J., et al., Cell, 2010. 141(2): p. 304-14.

[6] Yamada, M., et al., EMBO J, 2008. 27(19): p. 2471-83.

[7] Toropova, K., et al., Elife, 2014. 3. 